\title{
Application of PEI-Modified Magnetic Nanoparticles as Gene Transfer Vector for the Genetic Modification of Animals
}

\author{
Jinhui Cui, ${ }^{1}$ Haixin Cui, ${ }^{1}$ Yan Wang, ${ }^{1}$ Changjiao Sun, ${ }^{1}$ Kui Li, ${ }^{2}$ Hongyan Ren, ${ }^{2}$ and Wei Du ${ }^{1}$ \\ ${ }^{1}$ Research Center for Nanoscale Science in Agriculture, Institute of Environment and Sustainable Development in Agriculture, \\ Chinese Academy of Agricultural Sciences, Beijing 100081, China \\ 2 Institute of Animal Sciences, Chinese Academy of Agricultural Sciences, Beijing 100081, China \\ Correspondence should be addressed to Haixin Cui, haixincui@ieda.org.cn
}

Received 18 August 2011; Revised 16 November 2011; Accepted 20 November 2011

Academic Editor: Piao Liu

Copyright () 2012 Jinhui Cui et al. This is an open access article distributed under the Creative Commons Attribution License, which permits unrestricted use, distribution, and reproduction in any medium, provided the original work is properly cited.

To evaluate the performance of the magnetic nanoparticles as gene transfer vector for breeding transgenic animals, we investigated a new approach to deliver green fluorescent protein (GFP) gene to porcine kidney 15 (PK-15) and porcine embryonic fibroblast (PEF) cells using PEI-modified magnetic nanoparticles as gene vector. The morphology of the nanoparticles and nanoparticle/DNA complexes was characterized using scanning electron microscopy. It was found that the surface of the particles becomes coarse and rough with increased average diameter, which implied the effective conjugating between nanoparticles with DNA. The zeta potential of nanoparticle/DNA complexes drops down from $+29.4 \mathrm{mV}$ to $+23.1 \mathrm{mV}$ comparing with pure nanoparticles. Agarose gel electrophoresis experiments show that DNA plasmids can be protected effectively against degradation of exonuclease and endonuclease. The efficiency of gene delivery was affected by the mass ratio of nanoparticle/DNA and the amount of nanoparticle/DNA complexes. We confirm that the most optimal mass ratio of nanoparticle/DNA is $1: 1$ by conducting a series of experiments. This work provides important experimental basis for the application of the magnetic nanoparticles on gene delivery to porcine somatic cells, which is significant for the achieving of breeding new transgenic cloned pigs by using somatic cell nuclear transfer technique.

\section{Introduction}

Transgenic technology has been widely used in breeding new plant and animal varieties. Effective gene delivery into the target cells is an essential step in these practices. Both viral and nonviral vectors have been used for gene delivery. The viral vectors exhibit high delivery efficiency but immunogenicity, toxicity, high cost, oncogenic properties, and so forth [1-3]. Nonviral gene vectors, due to their reduced immunogenicity, improved safety and the ability to carry larger DNA loads and become an attractive alternate for gene delivery. Cationic liposomes have been widely used as nonvirus gene vectors [4-9], but, because of its cell-specific, easily inactivation of serum protein, and other reasons, its scope of application was also limited [10].

Because of the unique physical and chemical properties, nanoparticles have been widely used in the field of drug and gene delivery [11-13]. As a novel kind of gene vectors, nanoparticles have many advantages. Nanoparticles can protect DNA molecules against nuclease degradation by wrapping and condensing nucleic acids, realize the specificity of gene delivery by conjugating with special target molecules, and achieve controlled release of DNA effectively extending the duration of action [14]. Besides the advantages of ordinary nanoparticles, magnetic nanoparticles are superparamagnetic. They can induce the DNA moving and condensing to the target cells in the presence of a magnetic field, which can significantly improve their efficiency as gene carriers [15].

Although the application of magnetic nanoparticles in gene diagnosis and therapy has been studied extensively, the gene transfer systems based on magnetic nanoparticles for gene delivery into mammalian somatic cells are few studied. Effective gene delivery into mammalian somatic cells is an essential step in reproductive cloning using somatic cell nuclear transfer technique. Here, we report a new approach 
to deliver genes to porcine somatic cells using PEI-modified magnetic nanoparticles as gene vector. To investigate the potential of magnetic nanoparticles as gene transfer vectors in transgenic animal production, polyethyleneimine-(PEI) modified $\mathrm{Fe}_{3} \mathrm{O}_{4}$ magnetic nanoparticles were employed to transfer reporter gene into somatic cells, and the gene delivery efficiency was examined.

\section{Materials and Methods}

2.1. Plasmid DNA. The pEGFP-N1 plasmid, which expresses enhanced green fluorescent protein under the control of the cytomegalovirus (CMV) promoter, was purchased from $\mathrm{BD}$ Biosciences Clontech (Palo Alto, CA, USA). The plasmids DNA was amplified in Escherichia coli and purified using a Wizard Plus Maxipreps DNA Purification System (Promega) according to the manufacturer's protocols.

2.2. PEI-Modified Magnetic Nanoparticle/DNA Complex. PEI-modified $\mathrm{Fe}_{3} \mathrm{O}_{4}$ magnetic nanoparticles (Chemicell, German) were mixed well with pEGFP-N1 by certain mass ratio ( $1: 1$ for electron microscopy, zeta potential, and size measurement especially). The mixtures were incubated for $30 \mathrm{~min}$ at room temperature.

2.3. Electron Microscopy. For scanning electron microscopy (SEM), the PEI-modified $\mathrm{Fe}_{3} \mathrm{O}_{4} /$ EGFP-DNA complexes and PEI-modified $\mathrm{Fe}_{3} \mathrm{O}_{4}$ nanoparticles were ultrasonically dispersed in pure water, respectively. SEM micrographs were collected in JSM-6700F microscope operating at $5 \mathrm{kV}$.

2.4. Zeta Potential and Size Measurement. Nanoparticle zeta potential and size measurements were conducted using laser particle size analyzer (Malvern Zetasizer Nano ZS, England). Briefly, nanoparticles materials were suspended in pure water at a concentrate of $5 \mu \mathrm{g} \cdot \mathrm{mL}^{-1}$, and measurements were performed at $25^{\circ} \mathrm{C}$ and $2.0 \mathrm{~mm}$ measurement position for zeta potential and $5.5 \mathrm{~mm}$ measurement position for size distribution, respectively.

2.5. Combination and Protection Analysis of PEI-Modified Magnetic Nanoparticles and Plasmid DNA. In each experimental group, the weight of nanoparticles was $0.5 \mu \mathrm{g}$, and the concentration of plasmid DNA was $0.5 \mu \mathrm{g} \cdot \mu \mathrm{L}^{-1}$. The nanoparticles and pEGFP-N1 plasmid DNA were mixed at the mass ratio of $1: 1,1: 5,1: 10$, and $1: 20$ respectively, and then incubated for $30 \mathrm{~min}$ at room temperature. The combination results were measured by agarose gel electrophoresis ( $1 \% \mathrm{w} / \mathrm{v}$ agar, voltage: $90 \mathrm{~V}$, time: $30 \mathrm{~min})$. The DNA-nanoparticle complexes with restriction endonuclease EcoR I and exonuclease DNase Iat $37^{\circ} \mathrm{C}$ were digested for $1 \mathrm{~h}$, respectively. Agarose gel electrophoresis was used to gain results.

2.6. Cells and Culture. Porcine kidney-15 cells (PK15) were obtained from the American Type Culture Collection (Manassas, VA, USA). The cells were cultured at $37^{\circ} \mathrm{C}$ under a humidified atmosphere of $5 \% \mathrm{CO}_{2}$ and $95 \%$ air in Dulbecco's modified eagle medium (DMEM) supplemented with $10 \%$ fetal bovine serum (FBS), $0.1 \mathrm{mg} \cdot \mathrm{mL}^{-1}$ streptomycin sulfate, and $100 \mathrm{U} \cdot \mathrm{mL}^{-1}$ potassium penicillin G. Porcine embryonic fibroblast (PEF) were primary cell generously provided by H. Y. Ren (Institute of Animal Sciences, Chinese Academy of Agricultural Sciences, China); these cells were then cultured at $37^{\circ} \mathrm{C}$ under a humidified atmosphere of $5 \% \mathrm{CO}_{2}$ and $95 \%$ air in DMEM supplemented with $20 \%$ FBS.

2.7. Application of PEI-Modified Magnetic Nanoparticles for Gene Delivery to Porcine Somatic Cells. The green fluorescent protein (GFP) expression carrier (pEGFP-N1) was employed for report gene. If the GFP plasmid DNA can be delivered into the cells and exert its function efficiently, the green fluorescent protein will be synthesized by the expression of GFP gene and can be imaged by fluorescence microscope. PK-15 and PEF cells were used as the receptor cells. The cells were seeded in the 6-hole cell plate, incubated at $37^{\circ} \mathrm{C}$, $5 \% \mathrm{CO}_{2}$, and cultured until the confluency reached $70 \%-$ $80 \%$. Then PEI-modified magnetic nanoparticles were used to deliver pEGFP-N1 plasmid DNA to receptor cells. The nanoparticles were mixed with plasmid DNA at certain mass ratios, incubated at room temperature for $15 \mathrm{~min}$. Cells were rinsed twice with phosphate-buffered saline (PBS), and $1.5 \mathrm{~mL}$ DMEM with no serum and other supplement were added. After that, cells were incubated with nanoparticleDNA complexes on a $0.3 \mathrm{~T}$ permanent magnet at room temperature for $15 \mathrm{~min}$. After a further incubation of $5 \mathrm{~h}$, medium was replaced with DMEM supplemented with $10 \%$ FBS at $37^{\circ} \mathrm{C}, 5 \% \mathrm{CO}_{2}$. The expression of GFP gene in the receptor cells was observed with fluorescence microscope (Olympus) after $24 \mathrm{~h}$ of incubation. The cells with trypsin were harvested and washed twice with PBS. At least 10000 cells were acquired for flow cytometry (BD FACSAria).

2.8. Statistical Analysis. Each experiment without declaring was carried out for three times at the same condition. Results are reported as means \pm standard deviation. All statistical analyses were performed using the program Microsoft EXCEL 2003 from the Microsoft Corporation.

\section{Results and Discussion}

\subsection{Characterization of Nanoparticles and Nanoparticle/DNA Complexes}

3.1.1. Electron Microscopy. SEM images of both magnetic nanoparticles and nanoparticle/DNA complexes are shown in Figures 1(a) and 1(b). The pure nanoparticles are spherical shape with the average diameter about $150 \mathrm{~nm}$. As shown in Figure 1(b), the surface of nanoparticle/DNA complexes becomes coarse and rough, and the average diameter is also increased to $200 \mathrm{~nm}$, due to the formation of nanoparticle/DNA complexes and the adsorption of DNA on the surface of the nanoparticles. SEM results imply the effective conjugating property between nanoparticles and DNA. 


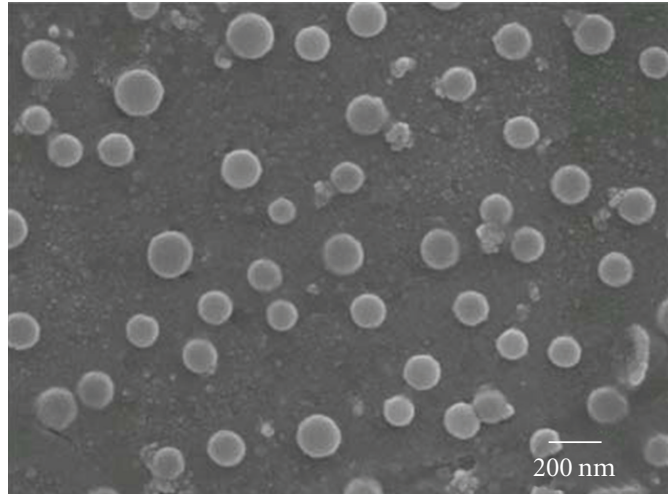

(a)

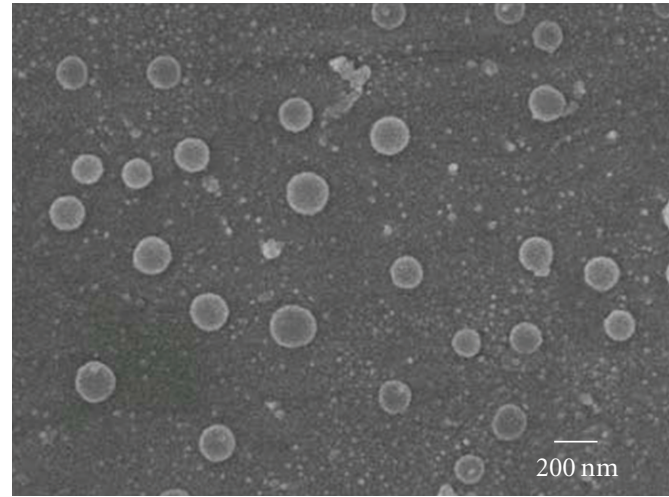

(b)

FIGURE 1: SEM images of magnetic nanoparticles and nanoparticle/DNA complexes: (a) SEM image of magnetic nanoparticles. The SEM image shows the spherical shape of the magnetic nanoparticles with diameter about $150 \mathrm{~nm}$. (b) SEM image of nanoparticle/DNA complexes. The nanoparticle/DNA complexes are also spherical but with rougher surface. The diameter of the complexes is about $200 \mathrm{~nm}$.

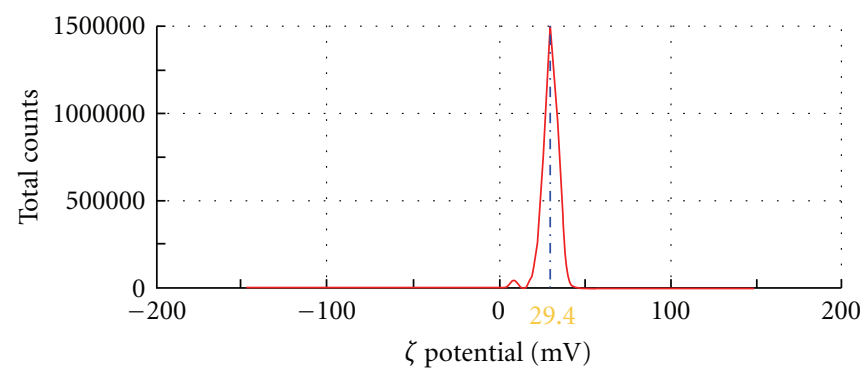

(a)

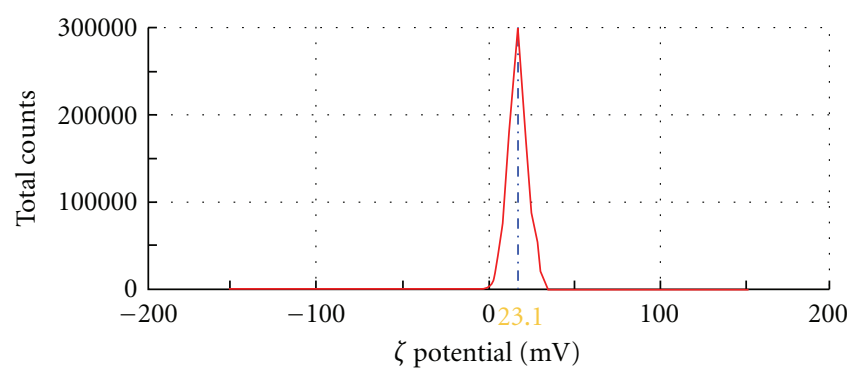

(b)

Figure 2: Zeta potential assay of nanoparticles and nanoparticle/DNA complexes. (a) The value at the peak of the curve shows that the Zeta potential of the nanoparticles is $+29.4 \mathrm{mV}$. (b) The Zeta potential of the nanoparticle/DNA complexes drops down to $+23.1 \mathrm{mV}$ compared with nanoparticles alone.

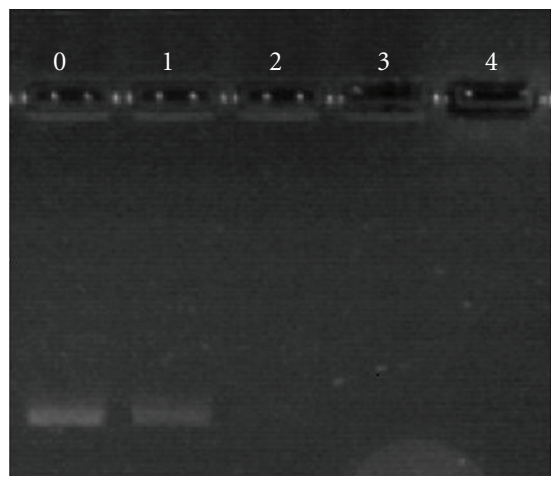

(a)

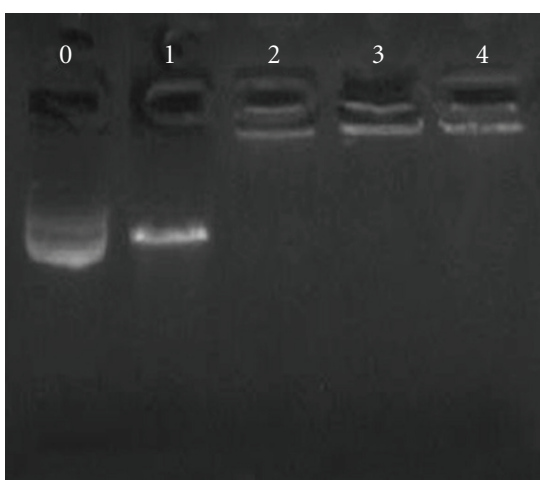

(b)

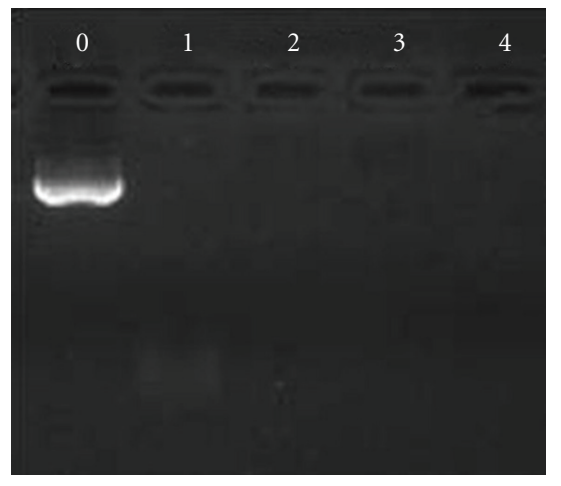

(c)

Figure 3: Agarose gel electrophoresis of plasmid DNA and nanoparticle/DNA complexes: (a) Lane 0: pure plasmid (control); lanes 1 4: nanoparticle/DNA complexes at the ratio of $1: 20,1: 10,1: 5$, and $1: 1$, respectively. (b) Lane 0: pure plasmid; lane 1: plasmid digested with EcoR I; lanes 2 4: nanoparticle/DNA complexes at the ratio of $1: 10,1: 5$, and 1:1, respectively, digested with EcoR I. (c) Lane 0: pure plasmid; lane 1: plasmid digested with DNase I; lanes 2 4: nanoparticle/DNA complexes at the ratio of $1: 10,1: 5$, and 1:1, respectively, digested with DNase I. 


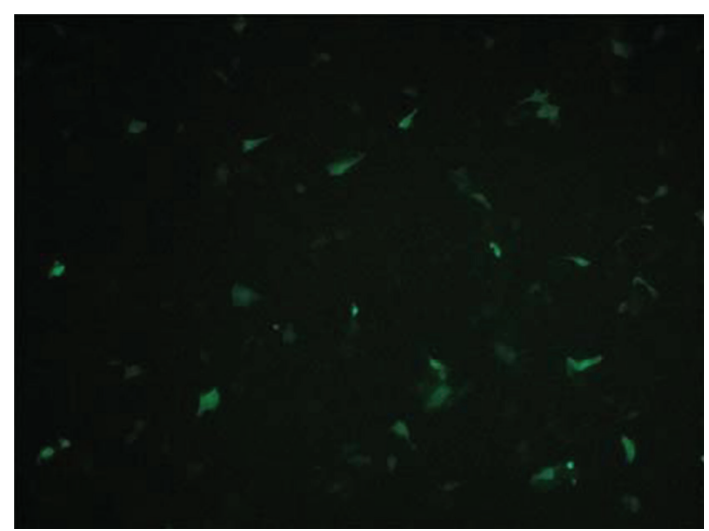

(a)

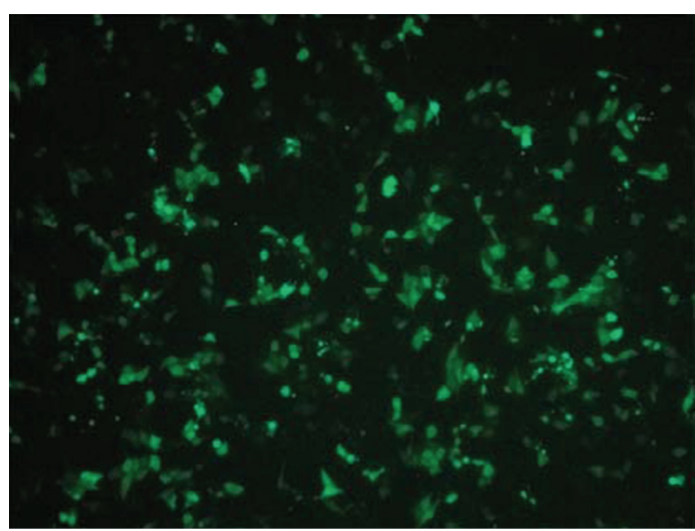

(b)

FIGURE 4: Fluorescent micrograph of the transfected PK-15: (a). Fluorescent micrograph of the PK-15 cells transfected without a magnetic field. (b) Fluorescent micrograph of the PK-15 cells transfected under a magnetic field. The results showed that the transfection efficiency can be significantly enhanced with magnetic field.

3.1.2. Zeta Potential and Size Measurement. The Zeta potential of the pure magnetic nanoparticles was positive charge of $+29.4 \mathrm{mV}$ (as shown in Figure 2(a)) with an average diameter of $(153.5 \pm 2.5) \mathrm{nm}$ (not shown) at neutral $\mathrm{pH}$. After conjugated with DNA to form complexes, the Zeta potential decreased to $+23.1 \mathrm{mV}$ (as shown in Figure 2(b)), and the average diameter increased to $(202.9 \pm 1.1) \mathrm{nm}$ (not shown). The results were consistent with the SEM morphology results. The decrease of the Zeta potential due to the negative charged DNA neutralized the positive charge of the nanoparticles.

3.2. Combination and Protection Analysis of PEI-Modified Magnetic Nanoparticles and Plasmid DNA. The binding and protecting ability of PEI-modified magnetic nanoparticles with plasmid DNA has been quantitatively investigated by using agarose gel electrophoresis. Agarose gel was performed in a $1 \%(\mathrm{w} / \mathrm{v})$ gel, ethidium bromide included for visualization for $30 \mathrm{~min}$ at $90 \mathrm{~V}$. Figure 3 (a) shows the photograph of the electrophoresis result for the nanoparticle/DNA complexes with different mass ratio of nanoparticle/DNA. The results showed that PEI-modified magnetic nanoparticles could bind the negative charged plasmid DNA, and the nanoparticles/DNA complexes retained at baseline (lane 2 4) because of the increased size of the complexes. As that the mass ratio of nanoparticle/DNA decreased to $1: 20$, free plasmid DNA was observed (lane 1), which illustrates the mass ratio of nanoparticle/DNA should be more than $1: 20$ in order to keep a complete combination between nanoparticles and plasmid DNA.

To investigate protecting ability of the nanoparticles for the DNA against enzyme degradation, $1 \mathrm{U}$ EcoR I and $1 \mathrm{U}$ DNase I were added to the pure plasmid DNA and nanoparticle/DNA complexes, respectively, and then incubated at $37^{\circ} \mathrm{C}$ for $1 \mathrm{~h}$. As shown in Figure 3(b) (lane 1), pure plasmid DNA was degraded by EcoR I, and linear DNA was produced. In the case of DNase I (Figure 3(c), lane 1), pure plasmid DNA was completely degraded into small fragments and hardly be visualized in the agarose gel. However, nanoparticle/DNA complexes were retained at the baseline, and no DNA strip was observed in the lane after being digested by EcoR I or DNase I (Figure 3(b), Figure 3(c), lane 2 4). These results showed that the magnetic nanoparticles can protect the plasmid DNA against enzyme degradation efficiently.

\subsection{Application of PEI-Modified Magnetic Nanoparticles for Gene Delivery to Porcine Somatic Cells. Based on the analysis of the PEI-modified magnetic nanoparticles, the combina- tion of the nanoparticles has a good ability to bind with plas- mid DNA. Moreover, the formation of nanoparticle/DNA complexes can protect DNA against enzyme degradation. Such advantages of the PEI-modified magnetic nanoparticles make it has a potential strength as vector for gene delivery. We further investigate the performance of the magnetic nanoparticles in the delivery system of PK-15 cells and PEF cells, respectively.}

3.3.1. Gene Delivery to PK-15 Cells. The nanoparticles were used to delivery pEGFP-N1 into PK-15 cells as gene vectors. The expression of GFP gene was detected by fluorescence microscope. It was found that the efficiency of GFP expression can be enhanced significantly in the presence of the magnetic filed (Figure 4). The reason is that the plasmid DNA can be directionally delivered to the receptor cells by magnetic nanoparticle under the magnetic field, which condensed the concentration of nanoparticle/DNA complexes on the surface of the cells, resulting in the increased cellular endocytosis of the complexes.

To investigate the effect of mass ratio of nanoparticle/DNA on the efficiency of gene delivery, the nanoparticle/DNA complexes were added to the receptor cells at the mass ratio of $2: 1,1: 1$, and $1: 2(2 \mu \mathrm{g}$ DNA, nanoparticles changed with the ratio). The results illustrate that the mass ratio of nanoparticle/DNA affects the gene delivery 


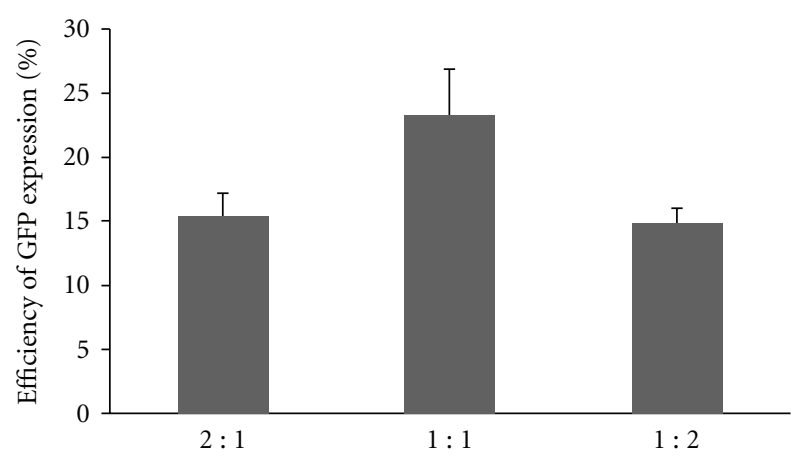

FIGURE 5: Expression efficiencies of GFP plasmid at different ratio of nanoparticles and plasmid DNA. The efficiency of GFP expression was influenced by the mass ratio of nanoparticles and plasmid DNA. Efficiencies are $(15.3 \pm 1.8) \%$ and $(14.8 \pm 1.2) \%$ at the mass ratio of $2: 1$ and $1: 2$, respectively. The highest gene delivery efficiency was obtained with $(23.3 \pm 3.5) \%$ at the nanoparticle/DNA mass ratio of $1: 1$. Shown are mean averages \pm standard deviation of three independent experiments. The differences are significant $(P<0.05)$ as determined by one-way ANOVA.

efficiency. When the mass ratios of nanoparticle/DNA are $2: 1$ and $1: 2$, the gene delivery efficiencies are $(15.3 \pm$ $1.8) \%$ and $(14.8 \pm 1.2) \%$, respectively (Figure 5$)$. The highest gene delivery efficiency was obtained with $(23.3 \pm 3.5) \%$ at the nanoparticle/DNA mass ratio of $1: 1$. It may be due to the cells viability decreased by the excess PEI on the surface of nanoparticles with too high nanoparticle ratio $(2: 1)$, resulting in the decreased efficiency. However, too low concentration of nanoparticles $(1: 2)$ could decrease the endocytosis effect of the complexes into the cells. It illustrates that the most optimal mass ratio of nanoparticle/DNA is $1: 1$ in order to keep a good performance in gene delivery experiments.

The efficiency of the gene delivery was also influenced by the amount of the nanoparticle/DNA complexes. The nanoparticle/DNA complexes were prepared with the mass ratio of $1: 1$, and then added to the cells with different amounts. The efficiencies of gene delivery with different amounts were shown in Figure 6. The efficiency was increased with the raised nanoparticle/DNA complexes in the low range of amounts, and then decreased with continuously increasing the amounts of nanoparticle/DNA complexes. The efficiency changed not obviously when the amounts of nanoparticle/DNA complexes were higher than $4 \mu \mathrm{g}$. The efficiency increased in the low range of complex amounts due to the dose effect. As the amount increased, excess complexes could lead to aggregation and increase the size of the complexes, which decreased the cellular endocytosis. The cell viability decreasing caused by the excess PEI of the nanoparticles was also the reason of efficiency decreasing.

3.3.2. Gene Delivery to PEF Cells. On the base of gene delivery into PK-15 cells, a preliminary study of gene delivery was also carried out in the system of PEF cells. The regulation of gene delivery to PEF cells was similar to PK-15 cells. The efficiency of gene delivery was the highest at the optimal

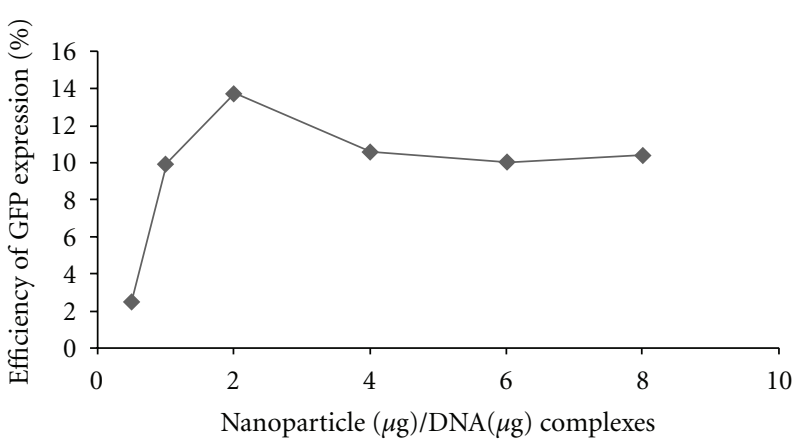

Figure 6: The efficiency curve of GFP expression with different amounts of nanoparticle/DNA complexes. The efficiency of GFP expression was influenced by the amount of the nanoparticle/DNA complexes. Efficiency increased with raised amount of complexes in low range, decreased with continuously increasing the amounts of complexes, and then changed not obviously when the complex amount surpassed $4 \mu \mathrm{g}$.

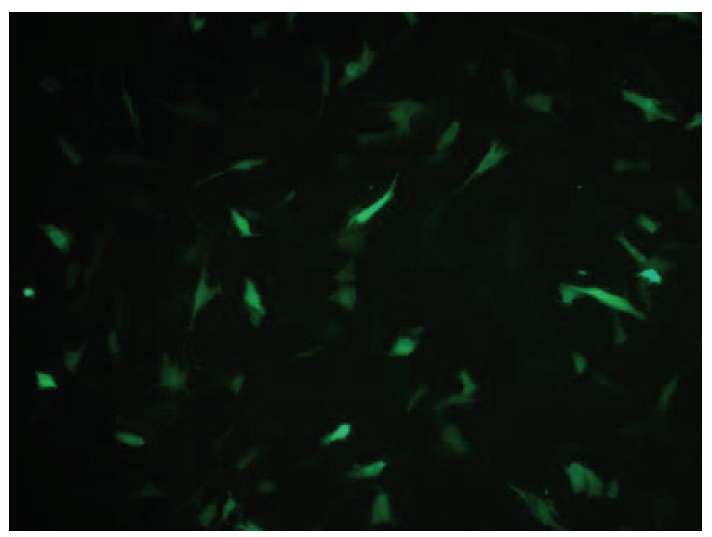

FIGURE 7: Fluorescent micrograph of GFP expression of PEF cells. The gene delivery into PEF cells was also carried out with PEImodified magnetic nanoparticles but still at a low level of efficiency compared with PK-15 cells.

mass ratio of $1: 1$ and changed with the amount of the nanoparticle/DNA complexes. But the efficiency was lower than that of PK-15 cells. The results showed that the PEImodified magnetic nanoparticles can also delivery GFP gene into PEF cells (Figure 7). The success delivery of GFP gene to PEF cells demonstrates that the PEI-modified magnetic nanoparticles can be employed for gene vector to deliver genes to porcine somatic cells, which may be significant for the achieving of breeding new transgenic cloned pigs using somatic cell nuclear transfer technique.

\section{Conclusions}

In conclusion, this work provides a new approach to deliver genes to porcine somatic cells using PEI-modified magnetic nanoparticles as gene transfer vector. The PEI-modified magnetic nanoparticles are spherical shape with the average diameter about $150 \mathrm{~nm}$. The surface of nanoparticle becomes coarse and rough, and the average diameter increases to 
$200 \mathrm{~nm}$ after conjugated with the plasmid DNA. The zeta potential of nanoparticle/DNA complexes drops down from $+29.4 \mathrm{mV}$ to $+23.1 \mathrm{mV}$ after the conjugation. Agarose gel electrophoresis experiments show that DNA plasmids can be loaded and protected effectively against degradation of exonuclease and endonuclease by PEI-modified magnetic nanoparticles. It is found that the efficiency of gene delivery is not only affected by the mass ratio of nanoparticle/DNA, but also the amount of the nanoparticle/DNA complexes.

The achieving of gene delivery into PK-15 and PEF cells by using magnetic nanoparticles provides an important experimental basis for the application of the magnetic nanoparticles as gene transfer vector for porcine somatic cells. It may provide a foundation study for the application of somatic cell nuclear transfer technique in breeding new transgenic cloned pigs.

\section{Acknowledgments}

This work was supported by National Transgenic Major Program (no. 2009ZX08010-006B) and Basic Scientific Research Fund of National Nonprofit Institutes (BSRF 201108).

\section{References}

[1] S. Lehrman, "Virus treatment questioned after gene therapy death," Nature, vol. 401, no. 6753, pp. 517-518, 1999.

[2] Y. Kaneda, "New vector innovation for drug delivery: development of fusigenic non-viral particles," Current Drug Targets, vol. 4, no. 8, pp. 599-602, 2003.

[3] A. Pathak, S. Patnaik, and K. C. Gupta, "Recent trends in nonviral vector-mediated gene delivery," Biotechnology Journal, vol. 4, no. 11, pp. 1559-1572, 2009.

[4] P. L. Felgner and G. M. Ringold, "Cationic liposome-mediated transfection,” Nature, vol. 337, no. 6205, pp. 387-388, 1989.

[5] E. G. Nabel, D. Gordon, Z. Y. Yang et al., "Gene transfer In Vivo with DNA-liposome complexes: lack of autoimmunity and gonadal localization," Human Gene Therapy, vol. 3, no. 6, pp. 649-656, 1992.

[6] N. J. Caplen, E. W. F. W. Alton, P. G. Middleton et al., "Liposome-mediated CFTR gene transfer to the nasal epithelium of patients with cystic fibrosis," Nature Medicine, vol. 1, no. 1, pp. 39-46, 1995.

[7] P. Jaroslav, G. E. Markus, G. Adam et al., "Optimization of nonviral transfection: variables influencing liposomemediated gene transfer in proliferating vs. quiescent cells in culture and in vivo using a porcine restenosis model," Journal of Molecular Medicine, vol. 80, no. 11, pp. 724-736, 2002.

[8] N. Kato, K. Nemoto, K. Nakanishi et al., "Nonviral HVJ (hemagglutinating virus of Japan) liposome-mediated retrograde gene transfer of human hepatocyte growth factor into rat nervous system promotes functional and histological recovery of the crushed nerve," Neuroscience Research, vol. 52, no. 4, pp. 299-310, 2005.

[9] S. Naoki, K. Chie, H. Atsushi et al., "Generation of highly potent nonviral gene vectors by complexation of lipoplexes and transferrin-bearing fusogenic polymer-modified liposomes in aqueous glucose solution," Biomaterials, vol. 29, no. 9, pp. 1262-1272, 2008.

[10] S. Y. Yang, J. S. Sun, C. H. Liu et al., "Ex vivo magnetofection with magnetic nanoparticles: a novel platform for nonviral tissue engineering," Artificial Organs, vol. 32, no. 3, pp. 195204, 2008.

[11] H. Mehrdad, A. Amir, and R. Pedram, "Hydrogel nanoparticles in drug delivery," Advanced Drug Delivery Reviews, vol. 60, no. 15, pp. 1638-1649, 2008.

[12] K. Avnesh, K. Y. Sudesh, and C. Subhash, "Biodegradable polymeric nanoparticles based drug delivery systems," Colloids and Surfaces B, vol. 75, no. 1, pp. 1-18, 2010.

[13] O. Veiseh, F. M. Kievit, J. W. Gunn, B. D. Ratner, and M. Zhang, "A ligand-mediated nanovector for targeted gene delivery and transfection in cancer cells," Biomaterials, vol. 30, no. 4, pp. 649-657, 2009.

[14] V. Vijayanathan, T. Thomas, and T. J. Thomas, "DNA nanoparticles and development of DNA delivery vehicles for gene therapy," Biochemistry, vol. 41, no. 48, pp. 14085-14094, 2002.

[15] S. W. Kamau, P. O. Hassa, B. Steitz et al., "Enhancement of the efficiency of non-viral gene delivery by application of pulsed magnetic field," Nucleic Acids Research, vol. 34, no. 5, article e40, 2006. 

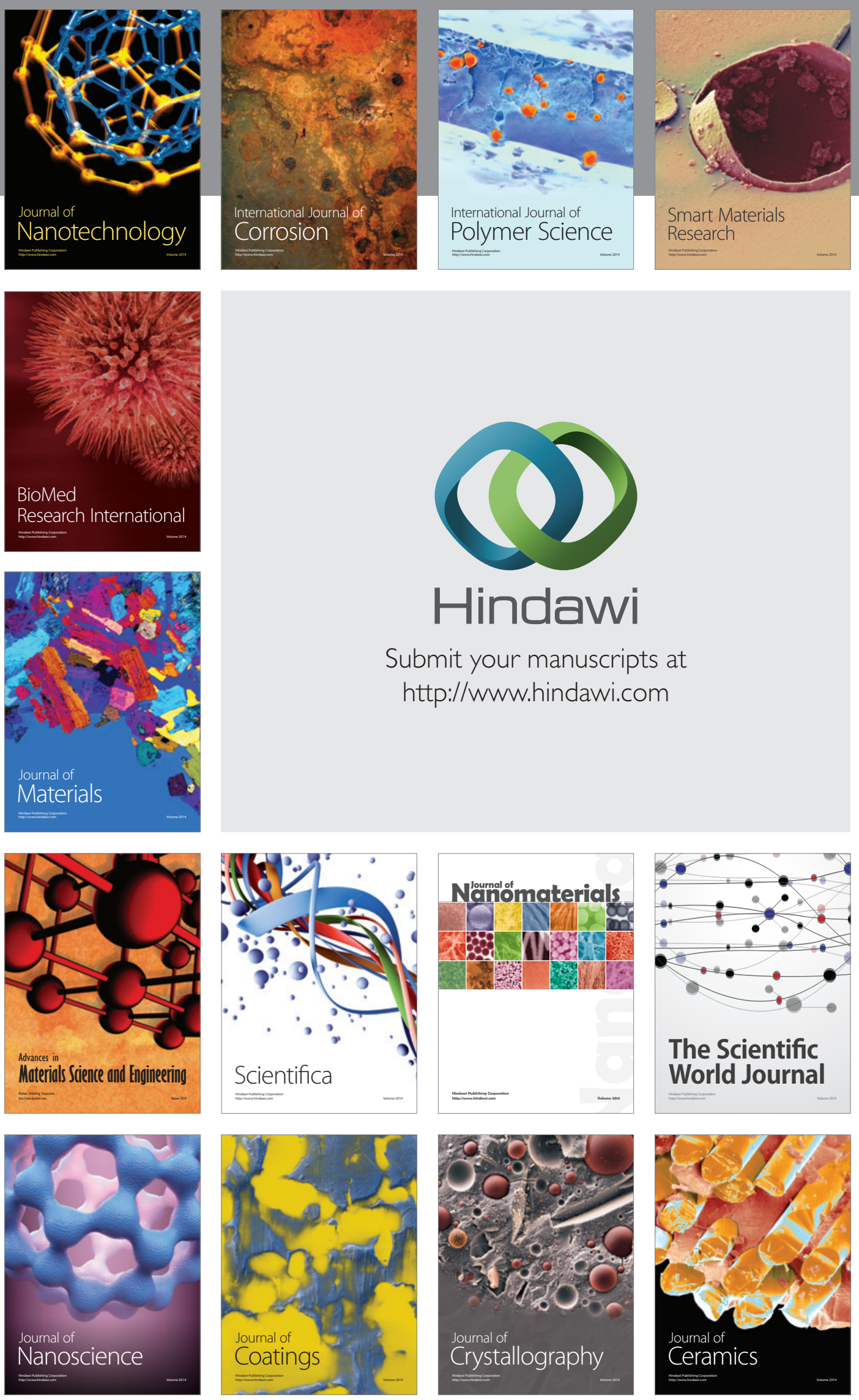

The Scientific World Journal

Submit your manuscripts at

http://www.hindawi.com

\section{World Journal}

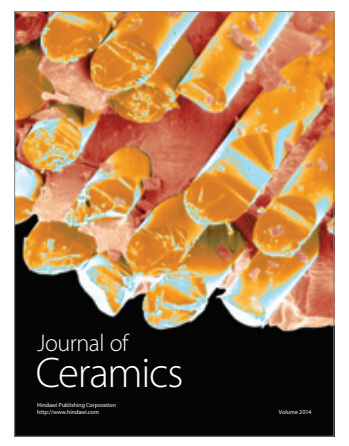

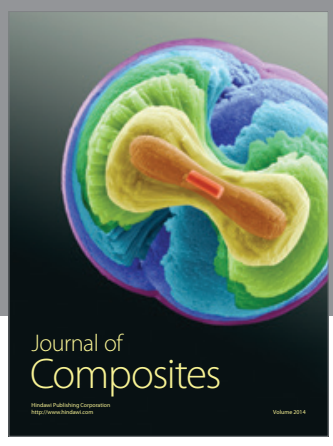
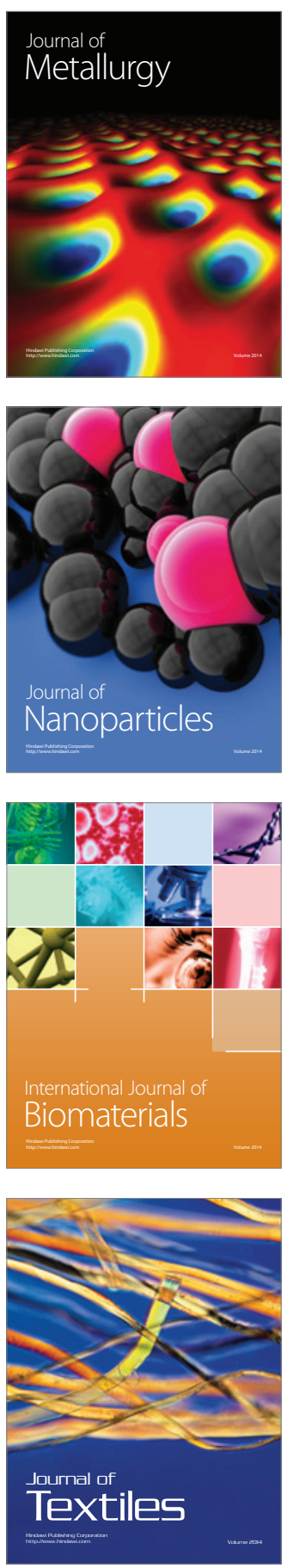